\title{
Migraña: efectividad del tratamiento quirúrgico. Revisión de la literatura
}

\author{
Nicolás Pereira C. ${ }^{1,2}$ y Jeffrey E. Janis ${ }^{3}$
}

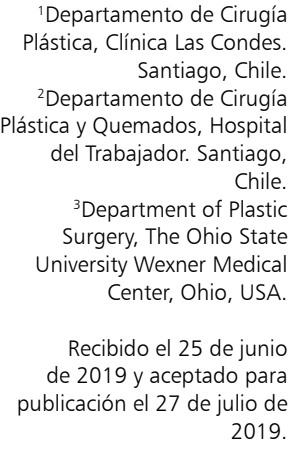

Correspondencia a:

Dr. Nicolás Pereira C. npereira@clinicalascondes.cl

\section{Migraine headache: effectiveness of surgical treatment. Review of the literature}

Migraine headaches affect a significant percentage of the population and the symptoms can interfere with quality of life in an important way. Despite advances in medical management, there is a proportion of patients who do not respond adequately to the pharmacological intervention. In recent years, new approaches have been proposed in the treatment of migraine. These are based on the theory that extracranial sensory branches of the trigeminal and cervical spinal nerves can become irritated, trapped or compressed at some point along their path, generating a cascade of physiological events that ultimately results in migraine. It has been shown that the diagnostic and therapeutic injection of botulinum toxin and the surgical decompression of these trigger points reduce or eliminate migraines in patients who do not respond adequately to the pharmacological intervention and remain symptomatic. The evidence supporting the efficacy and safety of surgical decompression of peripheral trigger points is rapidly accumulating, and the overall success rate of surgery approaches $90 \%$. This paper reviews the clinical evidence and aims to provide an article on the current state of the art in the surgical treatment of migraines.

Key words: migraine; surgical decompression; migraine surgery; trigger points.

\section{Resumen}

La migraña afecta a un porcentaje importante de la población y los síntomas pueden interferir con calidad de vida de manera importante. A pesar de los avances en el manejo médico, existe una proporción de pacientes que no responden adecuadamente a la intervención farmacológica. En los últimos años, se han planteado nuevos enfoques en el tratamiento de la migraña. Éstos se basan en la teoría que ramas sensoriales extracraneales del trigémino y de los nervios espinales cervicales pueden irritarse, atraparse o comprimirse en algún punto a lo largo de su trayecto, generándose una cascada de eventos fisiológicos que finalmente resulta en la migraña. Se ha demostrado que la inyección diagnóstica y terapéutica de toxina botulínica y la descompresión quirúrgica de estos puntos gatillos reducen o eliminan las migrañas en pacientes que no responden adecuadamente a la intervención farmacológica y siguen sintomáticos. La evidencia que respalda la eficacia y seguridad de la descompresión quirúrgica de los puntos de gatillos periféricos se está acumulando rápidamente, y la tasa de éxito general de la cirugía se acerca a $90 \%$. Este trabajo revisa la evidencia clínica y pretende proporcionar un artículo sobre el estado actual de la técnica en el tratamiento quirúrgico de las migrañas.

Palabras clave: migraña; descompresión quirúrgica; cirugía migraña; puntos gatillo.

\section{Introducción}

La migraña es un trastorno neurovascular que en ocasiones se trata de manera inadecuada con el tratamiento médico disponible, resultando en una discapacidad residual significativa. La Sociedad Internacional de Cefalea (IHS), la define como aquella que presenta al menos cinco episodios de cefalea, de 4 a 72 horas de duración y que cumplen con los criterios $\mathrm{A}_{\text {y }} \mathrm{B}^{1}$ :

A. Cefalea con al menos dos de cuatro de las siguientes características: Localización (unilateral); Tipo (pulsátil); Intensidad (moderada a severa); Agravantes (actividad física rutinaria). 
B. Durante la cefalea, al menos una de las siguientes características: Náuseas y/o vómitos; fotofobia y fonofobia.

Se habla de migraña crónica cuando los episodios de cefalea ocurren 15 o más días al mes, por más de tres meses, de los cuales 8 de los 15 episodios cumplen con los criterios de migraña descritos previamente ${ }^{1}$.

La migraña afecta al $12 \%$ de la población, siendo mucho más frecuente en mujeres que en hombres $(18 \% \text { versus } 6 \%)^{2}$. Los síntomas comúnmente interfieren con la funcionalidad y generan un gasto estimado de $\$ 1.000$ millones de dólares en costos médicos y $\$ 16.000$ millones en pérdida de productividad por año sólo en los Estados Unidos ${ }^{3}$, siendo la décimo segunda causa de discapacidad entre las mujeres en todo el mundo ${ }^{4}$. La mayoría de los pacientes con migraña requieren una combinación de medicamentos y conductas para controlar sus síntomas, lo que a menudo tiene una efectividad variable. Además, la terapia farmacológica estándar, que incluyen analgésicos profilácticos, tratamientos abortivo agudo y analgésico agudo, no están exentos de efectos secundarios que pueden impedir su uso ${ }^{5}$.

Tradicionalmente, la migraña se ha considerado como un fenómeno de etiología central. Sin embargo, recientemente se han identificado sitios gatillos extracraneales, cuya descompresión ha demostrado tener un impacto significativo en la sintomatología $^{6-13}$. Existe evidencia neurobiológica que apoya el concepto del desencadenante periférico como en el inicio de la migraña ${ }^{14}$. A través de microscopía electrónica y análisis proteómico, se han encontrado diferencias bioestructurales en la mielina de nervios periféricos resecados quirúrgicamente en pacientes con migraña comparados con nervios de pacientes sin migraña ${ }^{15}$.

Las primeras descripciones del tratamiento quirúrgico de la migraña se encuentran entre los años 100-200 dC donde Aretaeus recomendaba incisiones en los vasos sanguíneos de la cabeza para aliviar el dolor ${ }^{16}$. Con más detalle, el médico andaluz AlZahrawi describió hace 1.000 años aproximadamente, la técnica de cauterización de áreas del cráneo para aliviar el dolor de la migraña ${ }^{17}$. Seiscientos años después, el francés Ambroise Paré, rechazó este concepto y promovió la ligadura vascular para el tratamiento de la migraña ${ }^{18}$. El tratamiento de la anatomía vascular siguió su desarrollo y se complementó con un enfrentamiento más reciente de los nervios extracraneales. Martin et al., publicaron en 1964 el tratamiento quirúrgico de la neuralgia occipital mediante neurectomía ${ }^{19}$.
En los últimos años, los avances en neuroestimulación, como la estimulación occipital, la estimulación supraorbital transcutánea, la estimulación no invasiva del nervio vago y la estimulación transcraneal con corriente directa, han mostrado resultados prometedores en el tratamiento de la migraña crónica $^{20}$. Sin embargo, los estudios disponibles tienen una muestra pequeña, no se han replicado o poseen resultados negativos ${ }^{21}$.

Actualmente, el tratamiento quirúrgico de la migraña ha progresado. La inyección de toxina botulínica A (Botox ${ }^{\circledR}$ - Allergan, Dublín, Irlanda) ha sido aprobada por la FDA (Food and Drug Administration) como una terapia preventiva eficaz para la migraña crónica ${ }^{22-24}$. La descompresión quirúrgica o la neurectomía se ha utilizado en sitios seleccionados para lograr resultados a largo plazo, en los pacientes con compresión del nervio periférico ${ }^{6-13}$.

A continuación, presentamos una revisión de la literatura sobre la evidencia y efectividad de la cirugía en el tratamiento de la migraña.

\section{Tratamiento quirúrgico}

El tratamiento quirúrgico de la migraña actualmente incluye la descompresión de alguno de los siete sitios gatillos potenciales de compresión nerviosa, distribuidos en cuatro áreas periféricas principales (Tabla 1). Los pacientes se seleccionan luego de un diagnóstico de migraña crónica realizado por el neurólogo y/o tras el fracaso del tratamiento médico conservador. Las sesiones de quimiodenervación con Botox ${ }^{\circledR}$ permiten identificar y comprobar qué sitios gatillos se ven afecta$\operatorname{dos}^{7,9,10,12,13}$ y es un indicador predictivo positivo para el éxito quirúrgico ${ }^{24}$. Sin embargo, existe evidencia que sugiere que la presencia de una constelación de síntomas obtenidos mediante la historia clínica

Tabla 1. Sitios gatillos de compresión nerviosa y áreas periféricas principales

\begin{tabular}{|c|c|c|}
\hline \multicolumn{2}{|c|}{ Sitios gatillos } & Áreas \\
\hline I & Frontal (NSO/NST) & Frontal \\
\hline II & Cigomático temporal & Temporal \\
\hline III & Nasoseptal & Rinogénica \\
\hline IV & Occipital mayor & Occipital \\
\hline $\mathrm{V}$ & Auriculotemporal & Temporal \\
\hline VI & Occipital menor & Occipital \\
\hline VII & Numular & Variable \\
\hline
\end{tabular}


y un examen físico completo, es igual de eficaz para predecir qué sitios son susceptibles a ser tratados mediante descompresión quirúrgica ${ }^{25}$ (Tablas 2, 3, 4 y 5). Como alternativa, se puede realizar un bloqueo de nervio periférico diagnóstico con anestesia local para identificar los sitios gatillos susceptibles a descompresión. Adicionalmente, una tomografía computada y una evaluación con doppler manual son parte del estudio diagnóstico ${ }^{26}$. Las cuatro áreas principales incluyen la zona frontal, temporal, occipital y nasoseptal.

\section{Frontal}

Es producido por la compresión o irritación de los nervios supraorbitario (NSO) y supratroclear (NST), ramos de la división oftálmica del nervio trigémino (V1) (sitio I). Este sitio se aborda

\section{Tabla 2. Constelación de síntomas relacionados con la migraña} frontal

El dolor comienza sobre las cejas

El dolor usualmente comienza en las tardes

Existe una potente actividad de los músculos corrugadores que provocan arrugas en el entrecejo en reposo y actividad

Los puntos de emergencia de los NSO y NST del corrugador o del foramen están sensibles al tacto

Los pacientes tienen con frecuencia ptosis palpebral del lado afectado durante un episodio agudo

La presión sobre estos sitios puede abortar un episodio de migraña durante las etapas iniciales

La aplicación de compresas frías o calientes en estos sitios pueden aliviar el dolor

El dolor es de naturaleza implosivo

El estrés puede gatillar la migraña en este sitio

Tabla 4. Constelación de síntomas relacionados con la migraña rinogénica

El dolor comienza detrás de los ojos

El paciente frecuentemente despierta con dolor en la mañana o durante la noche

Con frecuencia, la migraña se gatilla con cambios de clima

El dolor puede estar acompañado de rinorrea del lado afectado

Este tipo de migraña puede estar relacionado con episodios de rinitis alérgica

Los ciclos menstruales pueden gatillar la migraña

El dolor usualmente se describe como explosivo

En la tomografía se pueden observar concha bulosa, desviaciones septales con contacto entre los cornetes y el septo, septo buloso, celdillas de Haller mediante la descompresión de la unidad miofascial, a través de la resección subtotal de los músculos corrugadores (MC) o la resección de todos los músculos glabelares que incluye los corrugadores, depresores de la ceja y procerus. Esto se puede realizar mediante un abordaje transpalpebral o endoscópico ${ }^{10}$, agregando una foraminotomía o fasciotomía de la escotadura supraorbitaria ${ }^{27}$.

\section{Temporal}

Se atribuye principalmente a la compresión nervio cigomático-temporal (NCT) (sitio II), ramo de la división maxilar del nervio trigémino (V2). El abordaje se puede realizar mediante la extensión de la incisión transpalpebral o vía endoscópica.

\section{Tabla 3. Constelación de síntomas relacionados con la migraña temporal}

El dolor empieza en la sien, aproximadamente $17 \mathrm{~mm}$ lateral y $6 \mathrm{~mm}$ craneal al canto lateral

Los pacientes usualmente despiertan con dolor luego de apretar los dientes durante la noche

Con frecuencia, se asocia con dolor de los músculos maséteros y temporal

Se puede observar desgaste de las fascetas dentales

La fricción o presión sobre el punto de salida del NCT en la fascia temporal profunda pueden abortar o reducir un episodio de migraña en las etapas iniciales

La aplicación de compresas frías o calientes en este sitio pueden aliviar el dolor

El dolor es de naturaleza implosivo

El estrés puede gatillar la migraña en este sitio

Tabla 5. Constelación de síntomas relacionados con la migraña occipital

El dolor comienza en el punto de salida del NOM en el músculo semiespinoso $(3,5 \mathrm{~cm}$ caudal a la tuberosidad occipital y $1,5 \mathrm{~cm}$ lateral a la línea media)

No hay un momento específico del día para el inicio del dolor

El paciente puede tener historia de latigazo cervical

Los músculos occipitales están usualmente rígidos

El ejercicio intenso puede gatillar la migraña

La presión sobre este sitio puede detener el dolor en etapas iniciales, mientras que en etapas más tardías el punto es doloroso

La aplicación de frío o calor en este sitio puede producir algún alivio del dolor

El estrés puede gatillar la migraña occipital 
Se realiza una neurectomía segmentaria o descompresión del NCT por debajo del nivel de la fascia temporal, sin haber diferencias significativas entre ambas técnicas ${ }^{28}$. Existe un grupo de pacientes que no responde a la descompresión de los cuatro puntos gatillos principales, o con migraña temporal aislada, lo cual puede estar provocado por la irritación del nervio auriculotemporal (NAT) (sitio V), ramo de la división mandibular del nervio trigémino (V3). El tratamiento del NAT se realiza mediante la ligadura de la arteria a través de una incisión pequeña en la zona del dolor y, cuando el nervio principal es el afectado, se agrega la neurectomía con neurotización del cabo proximal al músculo temporal.

\section{Occipital}

Se aborda mediante la descompresión del nervio occipital mayor (NOM) (sitio IV), ramo sensorial terminal de las ramas dorsales del segundo nervio espinal cervical (C2). Recientemente se ha propuesto que la compresión del nervio occipital menor (NOm) (sitio VI) y del nervio tercer occipital (NTO) también pueden contribuir al inicio de la migraña ${ }^{29}$. El NOM se aborda mediante una incisión en la línea media vertical o transversal, identificando la zona de compresión principal en la intersección del nervio con el músculo semiespinoso, Se debe realizar la descompresión hacia proximal hasta el nivel del músculo oblicuo inferior de la cabeza y resecar un segmento de músculo semiespinoso, medial y lateral al NOM. A distal se debe liberar la entrada al túnel del músculo trapecio, resecar la arteria occipital y liberar más allá de la línea nucal hasta el plano subcutáneo. Luego, se realiza un colgajo graso de base inferior que se ubica entre el músculo y el nervio para evitar el atrapamiento.

\section{Rinogénica}

En los pacientes con anomalías intranasales, como desviación o espolón septal, concha bullosa o hipertrofia de cornetes, se realiza septoplastía con o sin turbinectomía para eliminar los puntos de contacto mucoso aberrantes que pueden gatillar migraña.

\section{Evidencia clínica}

La efectividad del tratamiento quirúrgico de la migraña fue un hallazgo fortuito, luego de reportes anecdóticos de mejoría sintomática en pacientes que se sometieron a resección de los $\mathrm{MC}$ como parte de un procedimiento de rejuvenecimiento frontal. Guyuron et al. ${ }^{11}$, encuestaron a una población de pacientes para cuantificar esta observación y obtuvieron que de los 39 pacientes con migraña preexistente que se sometieron a resección de los $\mathrm{MC} ; 31(79,5 \%)$ experimentaron eliminación o mejoría significativa en sus migrañas. En respuesta a estos hallazgos, el mismo grupo realizó un estudio piloto prospectivo de cohorte en 22 pacientes que se sometieron a resección de los MC por vía transpalpebral o endoscópica con resección concurrente del NCT dependiendo de la respuesta preoperatoria a Botox $^{\circledR 10}$. En este estudio se encontró que el $95,5 \%$ presentó una eliminación completa o una mejoría significativa de sus migrañas (reducción de más de $50 \%$ en los síntomas), con un seguimiento medio de 347 días.

Dirnberger y Becker ${ }^{6}$, fueron los primeros en demostrar lo reproducible de la técnica, en un estudio de cohorte prospectivo que demostró la eliminación o mejoría significativa de la migraña en 41 de los 60 pacientes $(68,3 \%)$ que se sometieron a descompresión del sitio gatillo frontal, con una media de seguimiento de 12,8 meses. En un ensayo clínico controlado randomizado, se reclutaron 125 pacientes para tratamiento y control (4:1 respectivamente), en el cual se investigó la descompresión simultánea de los 4 puntos gatillo individualizada dependiendo de la respuesta a Botox $^{\circledR 7}$. De los pacientes que cumplieron con los criterios de seguimiento, $82 \mathrm{de}$ $89(92 \%)$ experimentaron eliminación o mejoría significativa de sus migrañas con un seguimiento promedio de 396 días, midiendo la gravedad de los síntomas con el Índice de Cefalea por Migraña (MHI - Migraine Headache Index), que combina la frecuencia, gravedad y duración de los episodios. Resultados similares se obtuvieron en una serie retrospectiva en la cual un $92,1 \%$ de los pacientes se benefició de la descompresión los sitios de gatillo frontal, temporal y occipital ${ }^{13}$. En 2009 Guyuron et al. ${ }^{9}$, realizaron un ensayo controlado randomizado con cirugía placebo, en el cual 41 de 49 pacientes $(83,7 \%)$ experimentaron mejoría significativa o eliminación de la migraña, estando 28 pacientes $(57,1 \%)$ en este último grupo. Esto fue significativamente mayor al grupo de cirugía placebo, donde 15 de 26 pacientes $(57,7 \%)(\mathrm{p}<0,05)$ reportaron mejoría parcial, y solo 1 paciente reportó eliminación de la migraña $(3,8 \%)(\mathrm{p}<0,001)$ después de 1 año de seguimiento. Los resultados son consistentes en el tiempo. Un estudio con seguimiento a 5 años reportó que, de los pacientes sometidos a cirugía descompresiva, un $88 \%$ experimentó una eliminación completa o una mejoría significativa de la migraña ${ }^{8}$.

En un estudio retrospectivo de 169 pacientes 
sometidos a cirugía descompresiva, se revisaron los factores que predicen el éxito o el fracaso quirúrgi$\mathrm{co}^{30}$. La cirugía era más exitosa cuando se abordan los 4 sitios gatillo $\mathrm{y}$, por el contrario, más probable que fallara cuando menos sitios de activación eran incluidos. El aumento del sangrado intraoperatorio también se asoció con el fracaso, ya que puede causar aumento de la inflamación y cicatrización, provocando un atrapamiento nervioso persistente después de la operación.

La migraña se describe comúnmente como un dolor pulsátil con un fuerte componente vascular en su etiología, existiendo una estrecha relación anatómica estrecha entre el NOM y la arteria occipital (AO). Se ha planteado la hipótesis que la irritación del NOM por la AO podría ser un factor que contribuya a la patogénesis de la migraña ${ }^{31}$. Sin embargo, en una serie de pacientes que se sometieron a la descompresión de NOM con o sin ligadura de la AO como parte del procedimiento, se evidenció que la ligadura de la $\mathrm{AO}$ no contribuyó al éxito de la descompresión del $\mathrm{NOM}^{32}$. Sin embargo, cabe señalar que en este estudio solo se resecó la AO cuando fue encontrada; si no se evidenciaba dentro del campo quirúrgico, ésta no se exploraba.

El NTO se sacrifica con frecuencia durante la descompresión del NOM. En una revisión retrospectiva, se compararon 111 pacientes con resección del NTO en contexto de la descompresión del NOM y 118 pacientes sin resección del NTO. Se encontró que la eliminación del NTO no afecta al éxito de la cirugía y no hubo diferencias entre ambos grupos, aunque las limitaciones de una revisión retrospectiva, incluida la variación en la técnica y la selección de los pacientes, podrían justificar un análisis prospectivo adicional ${ }^{33}$. En el mismo contexto, Ducic et al. ${ }^{34}$, describieron que la resección del NOM, en lugar de la descompresión, es una opción válida de tratamiento con éxito quirúrgico y alivio de la migraña en el $70,4 \%$ de los pacientes.

El punto gatillo frontal es el sitio más frecuente entre los pacientes que buscan tratamiento para la migraña mediante descompresión quirúrgica. La descompresión incompleta de gatillo frontal podría estar relacionada con la presencia de un foramen más que una escotadura supraorbital. De hecho, se estima que hasta un $25 \%$ de las personas tienen un foramen supraorbital. En un estudio, se compararon 2 grupos: resección de los músculos glabelares versus resección de los músculos glabelares más foraminotomía supraorbital, demonstrando que la resección muscular más foraminotomía supraorbital es superior, con una reducción significativa en el $M H I$ en comparación con la resección muscular aislada ${ }^{35}$.
Liu et al. ${ }^{36}$, compararon los abordajes endoscópicos y transpalpebral en el tratamiento del gatillo frontal, encontrando que el abordaje endoscópico tiene una tasa de éxito más alta. Los autores postulan que esto se puede deber a una resección más completa de los músculos corrugadores, especialmente el componente lateral, y a una visualización e identificación más fácil del foramen supraorbital y las ramas accesorias de NSO.

Recientemente se ha reportado el efecto de la inyección de grasa en el manejo de la migraña refractaria al tratamiento quirúrgico, obteniéndose una mejoría significativa de $69 \%$ y una resolución completa en $41,4 \%$ de los casos, con un seguimiento promedio de 29,4 meses $^{37}$.

\section{Conclusiones}

De acuerdo a la literatura disponible, la tasa promedio de éxito de la cirugía (eliminación o reducción de al menos 50\% de los síntomas), se ha acercado a casi el 90\%. Estos resultados han sido reproducidos por diferentes grupos y la mayoría de los estudios tienen un seguimiento mayor a 1 año mostrando un beneficio sostenido luego de la descompresión quirúrgica $^{38,39}$.

Los principales eventos adversos incluyen hipoestesia transitoria o parestesias en la zona operatoria, alopecía en la incisión, sangrado intraoperatorio controlado y movimiento asimétrico transitorio de las cejas. No se han reportado efectos secundarios importantes que hayan requerido reintervención. Esto sugiere que los efectos adversos relacionados con la descompresión quirúrgica son menos frecuentes y menos complejos que los efectos adversos que se pueden observar con la terapia médica tradicional. En 2011, Kung et al. ${ }^{40}$, revisaron el tratamiento médico actual para la migraña, incluida la eficacia y los efectos secundarios. En esta revisión, los autores destacan que, aunque algunos pacientes logran un alivio sostenido de los síntomas, un número considerable no pueden recibir o no son candidatos para el uso de estos medicamentos debido a sus comorbilidades. Este subgrupo de pacientes se beneficia más de la descompresión quirúrgica de los sitios gatillo.

Aparte de la importante mejoría en la calidad de vida que experimentan estos pacientes, el tratamiento quirúrgico de la migraña también es costo-efectivo. Aunque los costos iniciales de la cirugía pueden ser más altos, Faber et al. ${ }^{41}$, demostraron que, tanto los costos directos como los indirectos disminuyen después de la cirugía, con una reducción de costos 
total promedio de 3.949 dólares por año. Esto se debe a la reducción de los costos de medicamentos, menos consultas médicas y a una reducción de la cantidad de días laborales perdidos con la productividad recuperada.

La evidencia que respalda la desactivación quirúrgica de los nerviosos periféricos gatillo en la migraña está ampliamente descrita en la literatura. Cada vez más cirujanos están adoptando estas técnicas en su práctica ${ }^{42}$, lo cual genera la aparición de equipos multidisciplinarios compuestos por cirujanos plásticos, neurólogos y especialistas en manejo del dolor, que buscan en conjunto el beneficio de los pacientes para mejorar su calidad de vida.

La migraña afecta a un porcentaje importante de la población y los síntomas interfieren con la funcionalidad de los pacientes. La cirugía de migraña en pacientes seleccionados tiene una alta tasa de éxito. La evidencia actual respalda la desactivación quirúrgica de los sitios gatillo, lo cual, acompañado a un manejo multidisciplinario, permiten mejorar la calidad de vida de los pacientes.

\section{Responsabilidades éticas}

Protección de personas y animales. Los autores declaran que para esta investigación no se han realizado experimentos en seres humanos ni en animales.

Confidencialidad de los datos. Los autores declaran que en este artículo no aparecen datos de pacientes.

Conflicto de intereses: Jeffrey E. Janis, MD recibe derechos de autor de Thieme Publishing ${ }^{\circledR}$.

\section{Referencias}

1. Headache Classification Committee of the International Headache Society (IHS). The International Classification of Headache Disorders. 3rd edition (beta version). Cephalalgia 2013:33:629-808.

2. Stewart WF, Simon D, Shechter A, Lipton RB. Population variation in migraine prevalence: a meta-analysis. J Clin Epidemiol. 1995;48:269-80.

3. Goldberg LD. The cost of migraine and its treatment. Am J Manag Care 2005;11(2 Suppl):S62-S67.

4. Leonardi M, Steiner TJ, Scher AT, Lipton RB. The global burden of migraine: measuring disability in headache disorders with WHO's Classification of Functioning, Disability and Health (ICF). J Headache Pain 2005;6:429-40.

5. Whyte CA, Tepper SJ. Adverse effects of medications commonly used in the treatment of migraine. Expert Rev Neurother. 2009;9:1379-91.

6. Dirnberger F, Becker K. Surgical treatment of migraine headaches by corrugator muscle resection. Plast Reconstr Surg. 2004;114:652-7; discussion 658-9.

7. Guyuron B, Kriegler JS, Davis J, Amini SB. Comprehensive surgical treatment of migraine headaches. Plast Reconstr Surg. 2005;115:1-9.

8. Guyuron B, Kriegler JS, Davis J, Amini
SB. Five-year outcome of surgical treatment of migraine headaches. Plast Reconstr Surg. 2011;127:603-8.

9. Guyuron B, Reed D, Kriegler JS, Davis J, Pashmini N, Amini S. A placebocontrolled surgical trial of the treatment of migraine headaches. Plast Reconstr Surg. 2009; $124: 461-8$

10. Guyuron B, Tucker T, Davis J. Surgical treatment of migraine headaches. Plast Reconstr Surg. 2002;109:2183-9.

11. Guyuron B, Varghai A, Michelow BJ, Thomas T, Davis J. Corrugator supercilii muscle resection and migraine headaches. Plast Reconstr Surg. 2000;106:429-34; discussion 435-7.

12. Janis JE, Dhanik A, Howard JH. Validation of the peripheral trigger point theory of migraine headaches: singlesurgeon experience using botulinum toxin and surgical decompression. Plast Reconstr Surg. 2011;128:123-31.

13. Poggi JT, Grizzell BE, Helmer SD. Confirmation of surgical decompression to relieve migraine headaches. Plast Reconstr Surg. 2008;122:115-22; discussion 123-4.

14. Kosaras B, Jakubowski M, Kainz V, Burstein R. Sensory innervation of the calvarial bones of the mouse. J Comp Neurol. 2009;515:331-48.

15. Guyuron B, Yohannes E, Miller R, Chim H, Reed D, Chance MR. Electron microscopic and proteomic comparison of terminal branches of the trigeminal nerve in patients with and without migraine headaches. Plast Reconstr Surg. 2014;134:796e-805e.

16. Koehler PJ, van de Wiel TW. Aretaeus on migraine and headache. J Hist Neurosci. 2001;10:253-61.

17. Abū al-Qāsim Khalaf ibn 'Abbās alZahrāwī. Octauii Horatiani rerum medicarum lib. quatuor:Albucasis chirurgicorum omnium primarij, lib. tres; 1532. The Center for the History of Medicine, Countway Library of Medicine, Harvard University.

18. Ambroise Paré. The Works of Ambrose Parey, Chyrurgeon to Henry II, Francis II, Charles IX, and Henry III, Kings of France. London: J. Hindmarsh; 1691.

19. Martin BC, Fagan PJ. The surgical therapy of certain occipital headaches. Plast Reconstr Surg. 1964;33:266-8.

20. Halker Singh RB, Ailani J, Robbins MS. Neuromodulation for the Acute and Preventive Therapy of Migraine and Cluster Headache. Headache. 2019;59 Suppl 2:33-49.

21. Cho SJ, Song TJ, Chu MK. Treatment Update of Chronic Migraine. Curr Pain Headache Rep. 2017;21:26.

22. Aurora SK, Dodick DW, Turkel CC, DeGryse RE, Silberstein SD, Lipton RB, et al. OnabotulinumtoxinA for treatment of chronic migraine: results from the double-blind, randomized, placebocontrolled phase of the PREEMPT 1 trial. 
Cephalalgia 2010;30:793-803.

23. Diener HC, Dodick DW, Aurora SK, Turkel CC, DeGryse RE, Lipton RB, et al. OnabotulinumtoxinA for treatment of chronic migraine: results from the double-blind, randomized, placebocontrolled phase of the PREEMPT 2 trial. Cephalalgia 2010;30:804-14.

24. Lipton RB, Varon SF, Grosberg B, McAllister PJ, Freitag F, Aurora SK, et al. Onabotulinumtoxin A improves quality of life and reduces impact of chronic migraine. Neurology 2011;77:1465-72.

25. Liu MT, Armijo BS, Guyuron B. A comparison of outcome of surgical treatment of migraine headaches using a constellation of symptoms versus botulinum toxin type A to identify the trigger sites. Plast Reconstr Surg. 2012;129:413-9.

26. Guyuron B, Nahabet E, Khansa I, Reed D, Janis JE, et al. The Current Means for Detection of Migraine Headache Trigger Sites. Plast Reconstr Surg. 2015;136:8607.

27. Hagan RR, Janis JE, Falluco M. The Anatomical Morphology of the Supraorbital Notch: Clinical Relevance to the Surgical Treatment of Migraine Headaches. Plast Reconstr Surg. 2012;130:1227-33.

28. Guyuron B, Harvey D, Reed D. A Prospective Randomized Outcomes Comparison of Two Temple Migraine
Trigger Site Deactivation Techniques. Plast Reconstr Surg. 2015;136:159-65.

29. Dash KS, Janis JE, Guyuron B. The lesser and third occipital nerves and migraine headaches. Plast Reconstr Surg. 2005;115:1752-8; discussion 1759-60.

30. Larson K, Lee M, Davis J, Guyuron B. Factors contributing to migraine headache surgery failure and success. Plast Reconstr Surg. 2011;128:1069-75.

31. Janis JE, Hatef DA, Reece EM, McCluskey PD, Schaub TA, Guyuron B. Neurovascular compression of the greater occipital nerve: implications for migraine headaches. Plast Reconstr Surg. 2010;126:1996-2001.

32. Chmielewski L, Liu MT, Guyuron B. The role of occipital artery resection in the surgical treatment of occipital migraine headaches. Plast Reconstr Surg. 2013;131:351e-6e

33. Lee M, Lineberry K, Reed D, Guyuron B. The role of the third occipital nerve in surgical treatment of occipital migraine headaches. J Plast Reconstr Aesthet Surg. 2013;66:1335-9.

34. Ducic I, Felder JM III, Khan N, Youn S. Greater occipital nerve excision for occipital neuralgia refractory to nerve decompression. Ann Plast Surg. 2014;72:184-7.

35. Chepla KJ, Oh E, Guyuron B. Clinical outcomes following supraorbital foraminotomy for treatment of frontal migraine headache. Plast Reconstr Surg. 2012;129:656e-62e.

36. Liu MT, Chim H, Guyuron B. Outcome comparison of endoscopic and transpalpebral decompression for treatment of frontal migraine headaches. Plast Reconstr Surg. 2012;129:1113-9.

37. Guyuron B, Pourtaheri N. Therapeutic Role of Fat Injection in the Treatment of Recalcitrant Migraine Headaches. Plast Reconstr Surg. 2019;143:877-85.

38. Janis JE, Barker JC, Javadi C, Ducic I, Hagan R, Guyuron B. A Review of Current Evidence in the Surgical Treatment of Migraine Headaches. Plast Reconstr Surg. 2014;134:131s-41s.

39. American Society of Plastic Surgeons. Policy Statement: Migraine Headache Surgery. https://www.plasticsurgery.org/ Documents/HealthPolicy/Positions/ASPSStatement_Migraine-Headache-Surgery. pdf.

40. Kung TA, Guyuron B, Cederna PS. Migraine surgery: a plastic surgery solution for refractory migraine headache. Plast Reconstr Surg. 2011;127:181-9.

41. Faber C, García RM, Davis J, Guyuron B. A socioeconomic analysis of surgical treatment of migraine headaches. Plast Reconstr Surg. 2012;129:871-7.

42. Kung TA, Pannucci CJ, Chamberlain JL, Cederna PS. Migraine surgery practice patterns and attitudes. Plast Reconstr Surg. 2012;129:623-8. 